09

\title{
Спектрально-модуляционные характеристики лазеров с вертикальным резонатором
}

(C) М.И. Васьковская ${ }^{1,2}$, В.В. Васильев ${ }^{1,22,3}$, С.А. Зибров ${ }^{1,2}$, В.П. Яковлев ${ }^{3}$, В.Л. Величанский ${ }^{1,2,3,9}$

${ }^{1}$ ООО „Атомикс“, Москва, Сколково, Россия

${ }^{2}$ Физический институт им. П.Н. Лебедева РАН, Москва, Россия

${ }^{3}$ Национальный исследовательский ядерный университет „МИФИ“, Москва, Россия

ฯ E-mail: vlvlab@yandex.ru

Поступило в Редакцию 30 августа 2017 г.

Рассмотрены требования, предъявляемые к лазерам с вертикальным резонатором, в ряде задач метрологии, где используется оптическая накачка атомов щелочных металлов. Для лазеров различных производителей эти требования сопоставлены с экспериментально наблюдаемыми спектральными характеристиками при постоянном токе накачки и в режиме его модуляции СВЧ-сигналом. Показано, что сравнительно небольшое число лазеров позволяет в режиме СВЧ-модуляции получить спектр, требуемый для атомных часов на основе эффекта когерентного пленения населенностей.

DOI: 10.21883/PJTF.2018.01.45430.17019

Атомы щелочных металлов (прежде всего цезия, рубидия и калия) находят широкое применение в метрологии частоты и времени [1], магнитометрии [2] и гироскопии [3,4].

Диодные лазеры с вертикальным резонатором [5] особенно удобны для оптической накачки малогабаритных метрологических приборов, разрабатываемых для этих направлений. Важным достоинством таких лазеров при диаметре излучающей активной области $\leqslant 5 \mu \mathrm{m}$ является динамическая одномодовость излучения. Перечислим основные требования, предъявляемые к лазерам в названных приложениях.

1. Необходим одномодовый режим генерации линейно поляризованного излучения на фундаментальной поперечной моде со степенью 
подавления других поперечных мод и мод ортогональной поляризации не менее $20-30 \mathrm{~dB}$.

2. Ширина линии генерации не должна превышать величину порядка $100 \mathrm{MHz}$.

3. Центральная частота линии генерации лазера должна быть настроена на частоту атомного перехода с точностью не хуже $10 \mathrm{MHz}$, что в диапазоне длин волн $\sim 850 \mathrm{~nm}$ соответствует точности настройки длины волны $2.5 \cdot 10^{-5} \mathrm{~nm}$.

Поскольку достаточно настроить частоту излучения лазера на линию поглощения шириной порядка $1 \mathrm{GHz}$ и стабилизировать ее по центру линии с помощью системы обратной связи, допуск на частоту генерации лазера увеличивается на два порядка. Кроме того, допуск на частоту лазера тем больше, чем больше допустимый интервал рабочей температуры лазера. Если он составляет $3 \mathrm{~K}$, то требование к длине волны излучения становится еще на два порядка менее жестким $(0.25 \mathrm{~nm})$. Выполнение перечисленных статических требований необходимо для всех обсуждаемых приложений.

Перейдем к требованиям к динамическим характеристикам лазеров. В магнитометрии и гироскопии необходима только низкочастотная модуляция частоты излучения лазера для ее стабилизации по линии поглощения методом экстремального регулирования. Модуляция частоты излучения лазера, формирующая сигнал ошибки, осуществляется на частоте в диапазоне $1-10 \mathrm{kHz}$ при девиации частоты излучения порядка $100 \mathrm{MHz}$. Требуемая амплитуда модуляции тока не превышает $1 \mu \mathrm{A}$ при полном токе $\sim 1 \mathrm{~mA}$. Это не нарушает режим работы лазера и не накладывает дополнительных условий на требуемые характеристики.

К лазерам, используемым в малогабаритных стандартах частоты (наиболее массовое метрологическое применение) на основе когерентного пленения населенностей (КПН), предъявляются дополнительные требования. Ток лазера должен эффективно модулироваться на СВЧ-частоте, соответствующей половине сверхтонкого расщепления атомов ${ }^{133} \mathrm{Cs}(4.6 \mathrm{GHz})$ или ${ }^{87} \mathrm{Rb}(3.4 \mathrm{GHz})$. Важно, чтобы при этой модуляции сохранялись поляризация излучения и одномодовый режим генерации лазера. Появление других мод вызывает шум их разделения, уменьшает мощность рабочих компонент спектра и ухудшает отношение сигнал/шум.

В результате СВЧ-модуляции тока лазера в его спектре возникают боковые полосы, первые из которых являются рабочими. Они настра-

Письма в ЖТФ, 2018, том 44, вып. 1 
иваются в резонанс со сверхтонкими компонентами $D_{1}$-линии атомов. Для высокой долговременной стабильности такого стандарта частоты необходимо подавление световых сдвигов (т.е. зависимости частоты перехода 0-0 от световой мощности), возникающих из-за флуктуаций и дрейфа полной мощности лазера. Для подавления резонансных световых сдвигов необходимо равенство амплитуд полей в первых полосах, а для обеспечения требуемого отношения сигнал/шум эти амплитуды не должны быть слишком малы. Для подавления нерезонансных световых сдвигов увеличение частоты КПН резонанса из-за перекрестного воздействия первых полос и влияния поля несущей частоты должно компенсироваться ее уменьшением, обусловленным высшими боковыми полосами. В теории [6] показано, что баланс реализуется при такой мощности СВЧ-модуляции, когда амплитуда поля на несущей частоте близка к нулю (индекс модуляции $~ 2.4$ ).

Таким образом, спектр лазера в режиме модуляции должен удовлетворять следующим требованиям.

1. Мощности первых боковых полос должны составлять заметную долю от полной мощности лазера, поскольку они определяют уровень сигнала.

2. Мощность несущей и разность мощностей первых боковых полос должны быть минимальными.

3. Суммарной мощности высших боковых полос должно быть достаточно для компенсации нерезонансного светового сдвига.

Далее приведены результаты исследования спектральных характеристик лазеров, произведенных компаниями ULM, VIXAR, Princeton Optronics (PO) и в ФТИ им. А.Ф. Иоффе (далее „лазеры ФТИ“), в режиме СВЧ-модуляции тока. Одномодовость и тонкая структура спектра, связанная с поляризационным расщеплением мод, регистрировались с помощью интерферометра или атомной ячейки. Подробнее установка описана в [7]. Установлено, что во всех типах лазеров при допустимых токах накачки генерация происходит только на одной (по трем индексам) моде. На рис. 1 показаны ватт-амперная характеристика и спектр пропускания интерферометра, полученные при линейном сканировании тока и частоты лазера ФТИ. Видно, что одномодовый режим реализуется начиная от порога и сохраняется во всей области допустимых токов (до $3 \mathrm{~mA}$ ). Однако не во всех лазерах всегда доминирует одна поляризация. Наиболее часто неустойчивость поляризации наблюдалась у лазеров VIXAR и PO, реже для лазеров ULM. На рис. 2, $a$ приведены

Письма в ЖТФ, 2018, том 44, вып. 1 


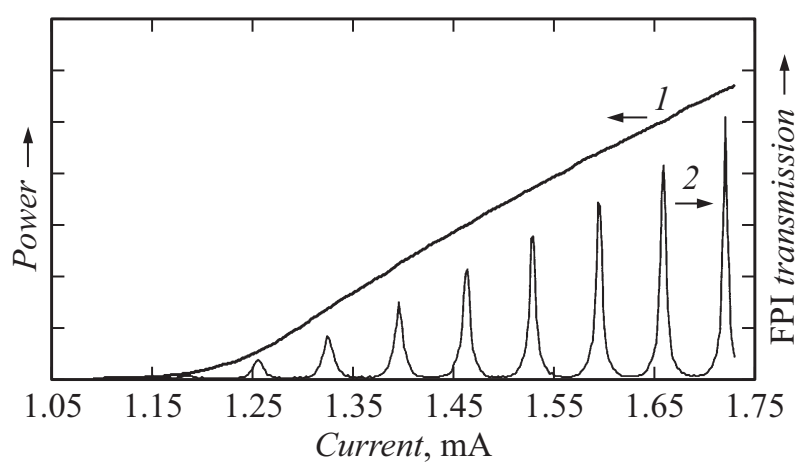

Рис. 1. Ватт-амперная характеристика лазера ФТИ (1) и резонансы пропускания интерферометра Фабри-Перо (FPI) (2) с областью свободной дисперсии $24 \mathrm{GHz}$.

спектры поглощения излучения лазера ULM на $D_{1}$-линии ${ }^{87} \mathrm{Rb}$ для трех положений поляризатора. При угле поворота поляризатора, принятом за $0^{\circ}$, наблюдается спектр 1 . Он прописан основной модой. При угле поворота $90^{\circ}$ спектр 2 регистрируется побочной модой ортогональной поляризации. Для промежуточного угла поляризатора (спектр 3) оба спектра наблюдаются одновременно. Таким образом, генерация происходит одновременно на двух ортогонально поляризованных модах. Спектральное расщепление этих мод составляет примерно $3.5 \mathrm{GHz}$, что согласуется с данными работы [8]. Это не противоречит паспортным данным лазера, поскольку степень подавления моды ортогональной поляризации гарантирована и наблюдается для рабочего тока $2 \mathrm{~mA}$. Отметим, что лазеры ФТИ [9] имеют высокую степень подавления моды ортогональной поляризации практически во всем диапазоне допустимых токов. Из-за малой разности потерь ортогонально поляризованных мод их переключение может быть вызвано изменением не только температуры/тока, но и даже частоты СВЧ-модуляции. На рис. $2, b$ показано влияние частоты СВЧ-модуляции на переключение мод ортогональных поляризаций (лазер ULM из той же серии). Видно, что ток, при котором происходит подавление моды $S$ с одновременным увеличением мощности моды с ортогональной поляризацией $M$, зависит от частоты СВЧ-модуляции. При меньших значениях тока моды сосуществуют и

Письма в ЖТФ, 2018, том 44, вып. 1 

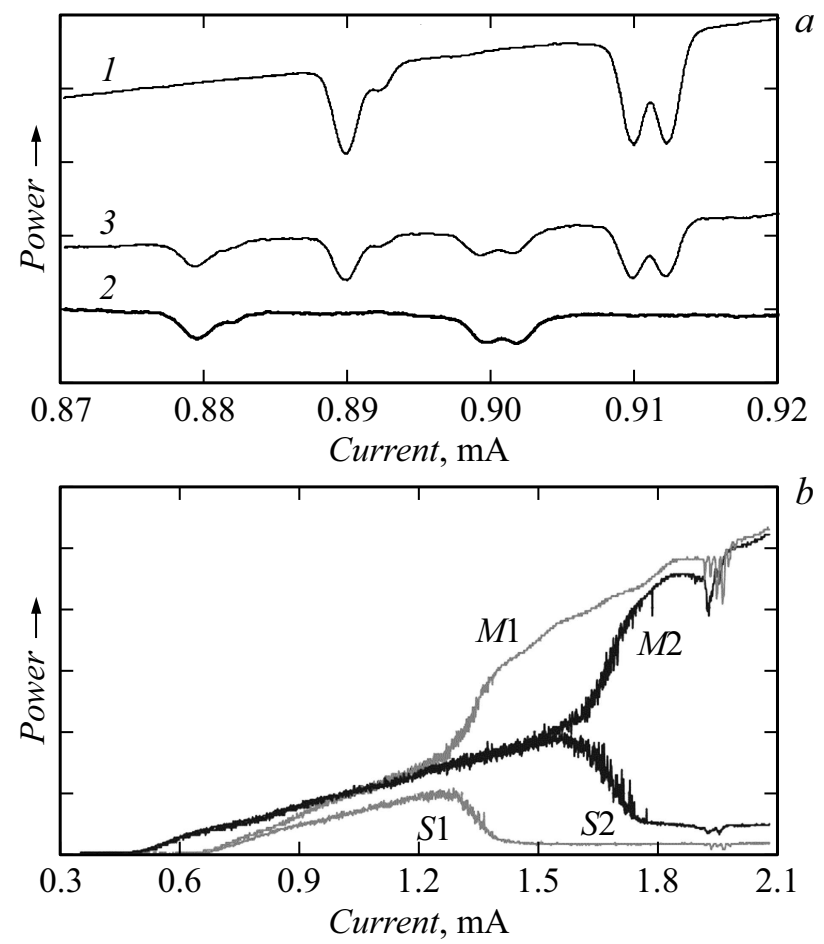

Pис. 2. Проявление побочной поляризационной моды в лазерах ULM. $a$ ) линия поглощения ${ }^{87} \mathrm{Rb}$, прописанная излучением основной $(1)$, побочной моды (2) и обеих мод одновременно (3) при сканировании тока лазера; $b-$ ватт-амперная характеристика излучения для основной $(M)$ и побочной $(S)$ мод в режиме модуляции тока накачки для двух частот модуляции: $M 1, S 1-3 \mathrm{GHz}, M 2$, $S 2-1 \mathrm{GHz}$.

близки по амплитуде. Подавление одной из мод при больших токах не является полным.

На рис. 3 приведены спектры излучения лазеров в режиме СВЧмодуляции тока при фиксированном значении его средней величины. Здесь в отличие от рис. 1 сканируется частота резонанса интерферометра. Во всех случаях область свободной дисперсии интерферометра превышала ширину регистрируемых спектров. Сопоставим их с пере-

Письма в ЖТФ, 2018, том 44, вып. 1 
56

М.И. Васьковская, В.В. Васильев...

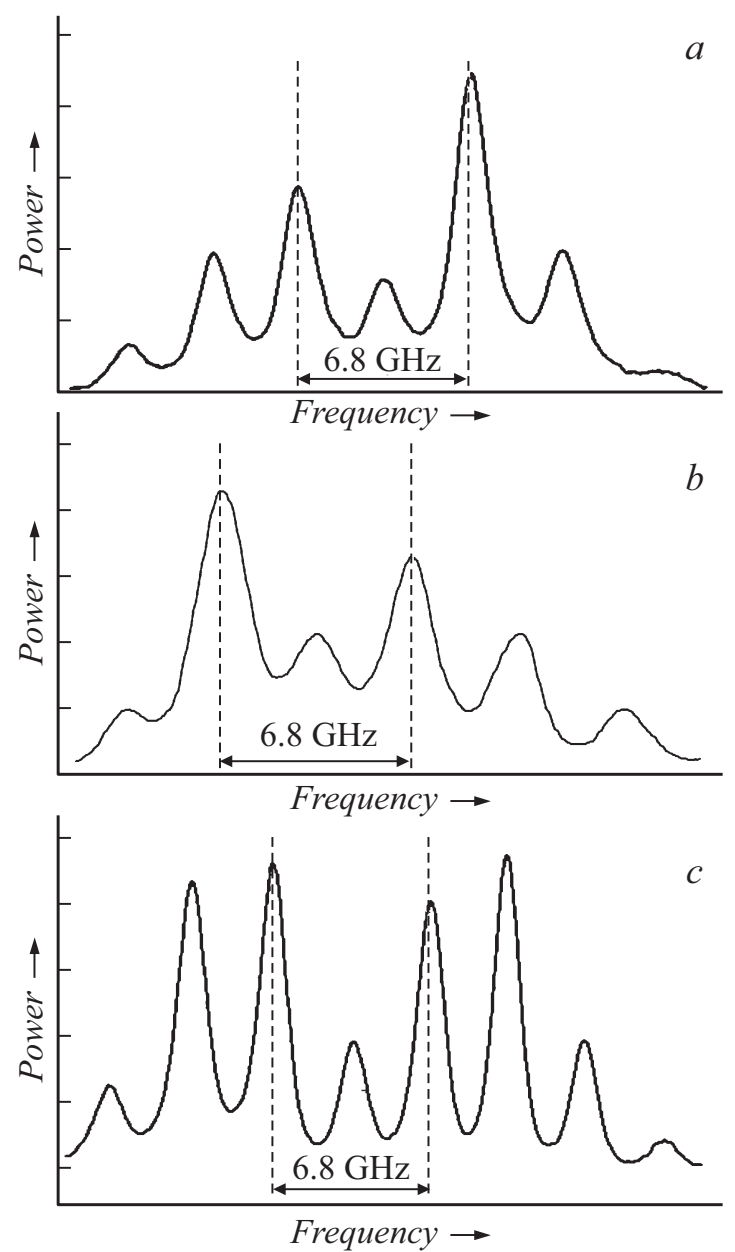

Pис. 3. Спектры излучения различных лазеров в режиме модуляции тока на частоте $3.4 \mathrm{GHz} . a$ - VIXAR, $b$ - лазеры ФТИ, $c$ - ULM. Показан частотный интервал между первыми боковыми компонентами.

численными выше требованиями. На основе приведенных спектров и других многочисленных измерений в диапазоне частот модуляции тока

Письма в ЖТФ, 2018, том 44, вып. 1 
$1-5 \mathrm{GHz}$ установлено, что амплитуда несущей компоненты спектра в исследованных лазерах никогда не уменьшается до нуля, что должно происходить при идеальной частотной модуляции с индексом 2.4. Для большинства лазеров амплитуды первых рабочих боковых полос не равны (рис. 3,a,b). Характер асимметрии для первых и вторых боковых полос, как правило, противоположен. Тем не менее среди лазеров (ULM) одной серии есть образцы $(\sim 10 \%)$ с близкими амплитудами первых боковых полос. Примерно таков же процент лазеров, в которых отрицательный световой сдвиг от вторых боковых полос компенсирует положительный сдвиг от несущей и первых боковых полос. Спектр излучения лазера, при котором реализуется такой баланс, показан на рис. 3,c. В остальных лазерах при увеличении индекса модуляции либо еще больше проявляется асимметрия спектра, либо происходит перераспределение мощности лазера по большому числу боковых полос с недопустимым падением уровня полезного сигнала.

Таким образом, по статическим характеристикам бо́льшая часть исследованных лазеров перечисленных производителей удовлетворяет предъявляемым требованиям и может использоваться в магнитометрах и гироскопах. Лишь незначительная часть лазеров не подходит изза нестабильности поляризации, особенно при низких токах накачки. В случае модуляционных спектров ситуация обратная: лишь немногие лазеры удовлетворяют требованиям, предъявляемым к ним в атомных часах на основе КПН-эффекта.

Известно, что лазеры с вертикальным резонатором имеют низкую стоимость, пока не требуется, чтобы длина волны генерации была задана с точностью до нескольких единиц четвертого знака. Однако изза трудно устранимых остаточных градиентов температуры в процессе роста лазерных структур на пластине большого диаметра выход таких лазеров очень мал, что резко увеличивает их стоимость. Поэтому дополнительная отбраковка части таких лазеров по спектральномодуляционным характеристикам крайне нежелательна. В связи с этим требуется проведение дальнейших экспериментальных и теоретических исследований указанных модуляционных характеристик. Возможными параметрами, влияющими на асимметрию спектра, являются сдвиги частоты генерации относительно вершины линии усиления и, что более вероятно, относительно спектрального максимума коэффициента отражения брэгговских зеркал. Следует отметить, что модуляция тока лазера приводит к модуляции не только частоты, но и мощности

Письма в ЖТФ, 2018, том 44, вып. 1 
излучения лазера. Неизбежное присутствие амплитудной модуляции с неконтролируемым сдвигом фазы относительно частотной модуляции и нелинейное взаимодействие спектральных компонент в излучении лазера не позволяют пока адекватно теоретически описать спектр при большом индексе модуляции. Для малых индексов модуляции это было проделано в работе [7].

В заключение отметим, что лазеры ФТИ являются первыми лазерами с вертикальным резонатором, удовлетворяющими метрологическим требованиям, полностью разработанными в России. Уже сейчас их характеристики (возможность настройки на атомную линию, динамическая одночастотность) сравнимы с характеристиками лучших зарубежных лазеров этого типа.

\section{Список литературы}

[1] Knappe S. // Comprehensive Microsystems. 2007. V. 3. P. 571-612.

[2] Knappe S., Kitching J. // Optical magnetometry/ Eds D. Budker, D.F.J. Kimball. Cambridge University Press, 2013. P. 125-141.

[3] Donley E.A., Kitching J.E. // ibid. P. 369-386.

[4] Козлов А.Н., Зибров С.А., Шутов В.В., Васильев В.В., Вершовский А.К., Пазгалёв А.С., Величанский В.Л. // Успехи современной радиоэлектроники. 2013. № 12. C. 43-59.

[5] Michalchic R. // VCSELs: Fundamentals, technology and applications of verticalcavity surface-emitting Lasers / Ed. R. Michalzik. Berlin: Springer-Verlag, 2013. P. 19-75.

[6] Zhu M., Cutler L.S. // Proc. of the 32nd annual precise time and time interval systems and applications meeting / Ed. L.A. Breakiron. US Naval Observatory, 2001. P. 311.

[7] Васьковская М.И., Васильев В.В., Зибров С.А., Величанский В.Л., Акимова И.В., Богатов А.П., Дракин А.Е. // Квантовая электроника. 2017. Т. 47. № 9. С. $835-841$.

[8] Panajotov K., Prati F. // VCSELs: Fundamentals, technology and applications of vertical-cavity surface-emitting lasers / Ed. R. Michalzik. Berlin: Springer-Verlag, 2013. P. 181-231.

[9] Nazaruk D.E., Blokhin S.A., Maleev N.A., Bobrov M.A., Kuzmenkov A.G., Vasil'ev A.P., Gladyshev A.G., Pavlov M.M., Blokhin A.A., Kulagina M.M., Vashanova K.A., Zadiranov Yu.M., Fefelov A.G., Ustinov V.M. // J. Phys. Conf. Ser. 2014. V. 572. P. 012036. (1-6).

Письма в ЖТФ, 2018, том 44, вып. 1 